\title{
Romance and sex before marriage among young women and men in Jharkhand
}

International Institute for Population Sciences (IIPS)

Population Council

Follow this and additional works at: https://knowledgecommons.popcouncil.org/departments_sbsr-pgy

Part of the Demography, Population, and Ecology Commons, Family, Life Course, and Society Commons, International Public Health Commons, and the Medicine and Health Commons How does access to this work benefit you? Let us know!

\section{Recommended Citation}

International Institute for Population Sciences (IIPS) and Population Council. 2009. "Romance and sex before marriage among young women and men in Jharkhand," Youth in India: Situation and Needs Policy Brief no. 10. Mumbai: IIPS. 


\section{Romance and sex before marriage among young women and men in Jharkhand}

Pre-marital partnerships among youth, including those that do not involve sexual relations, are widely discouraged in India. Indeed, there is a widespread perception that youth do not find opportunities to mix and form romantic relationships and that pre-marital sex is negligible. These kinds of perceptions are often cited to argue against the provision of sex or family life education and sexual and reproductive health services to unmarried young people.

While several small and unrepresentative studies have noted that sizeable proportions of young people, particularly young men, are sexually active before marriage, ${ }^{\text {a } c d e f}$ the lack of rigorous and representative evidence on the levels and patterns of intimate partnerships among youth continues to hamper designing of appropriate policies and programmes to address youth sexual health needs. This policy brief documents the extent of pre-marital romantic and sexual relations experienced by young men and women in Jharkhand, and the extent to which pre-marital sexual experiences among young people are informed, safe and wanted.

The study

Data are drawn from the Youth in India: Situation and Needs study, a sub-nationally representative study undertaken for the first time in India of key transitions experienced by young people in six states of India. The study included a representative survey of young people in both rural and urban settings. Respondents included unmarried women and men and married women aged 15-24 and, in view of the paucity of married men in these ages, married men aged 15-29.

In Jharkhand, the survey was conducted in 2006. A total of 8,814 married and unmarried young women and men were interviewed in the survey. These included 2,684 married young women, 2,730 unmarried young women, 1,259 married young men and 2,141 unmarried young men. This brief is based on data obtained from 2,637 young men and 5,414 women aged 15-24.

Acknowledging that young people may have been reluctant to disclose pre-marital sexual experiences, the Youth Study included a number of approaches to elicit data on sexual behaviour. Questions were posed both in a face-to-face interview format as well as an anonymous format in which youth were asked to mark a blank card indicating whether or not they had ever experienced pre-marital sex, place the card in an envelope, seal it and return it to the interviewer. Envelopes were opened only at study headquarters. In addition, youth were also asked to report, anonymously, the pre-marital sexual experiences of their peers.

\footnotetext{
a Abraham, L. 2001. "Redrawing the lakshman rekha: Gender differences and cultural constructions in youth sexuality in urban India," South Asia 24:133-56.

b Abraham, L. 2002. "Bhai-behen, true love, time pass: Friendships and sexual partnerships among youth in an Indian metropolis," Culture, Health, and Sexuality 4(3):337-53.

c Abraham, L. and K.A. Kumar. 1999. "Sexual experiences and their correlates among college students in Mumbai city, India," International Family Planning Perspectives 25(3):139-46.

d Alexander, M., L. Garda, S. Kanade et al. 2006a. Formation of Partnerships among Young Women and Men in Pune District, Maharashtra. New Delhi: Population Council

e Alexander, M., L. Garda, S. Kanade et al. 2006b. "Romance and sex: Pre-marital partnership formation among young women and men, Pune district, India," Reproductive Health Matters 14(28):144-55.

${ }_{\mathrm{f}}$ Awasthi, S., M. Nichter and V.K. Pande. 2000. "Developing an interactive STD prevention programme for youth: Lessons from a north Indian slum," Studies in Family Planning 31(2):138-50.
} 
Opportunities to form pre-marital romantic relationships do exist for young people Despite norms prohibiting pre-marital opposite-sex mixing, findings indicate that opportunities to form pre-marital romantic relationships did exist for young people. Findings suggest that $27-28 \%$ of young men and women had either been approached by or had approached a person of the opposite sex to form a romantic liaison. Moreover, $22 \%$ of young men and $14 \%$ of young women acknowledged the experience of a romantic partnership before marriage. Where romantic relationships occurred, they were usually hidden from parents but not from peers. Among those who had experienced a pre-marital romantic partnership, over $75 \%$ of young men and women reported that their peers were aware of their relationship; however, only $28 \%$ of young men and $42 \%$ of young women reported that their parents were aware of their relationship.

Extent of pre-marital romantic relationships: percentage of youth who reported a romantic partner

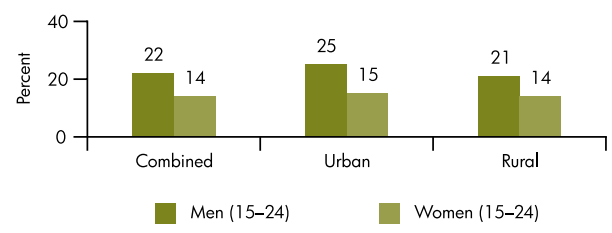

The majority of young men and women who reported a pre-marital romantic partnership had engaged in a range of intimate behaviours with the romantic partner, from holding hands to kissing on the lips to sexual intercourse. Over $90 \%$ of young men and $80 \%$ of young women had held hands with a romantic partner; however, consistently fewer reported each progressively more intimate behaviour, including hugging, kissing and engaging in sex. Even so, it is notable that young women were as likely as young men to report such intimate behaviours as kissing and engaging in sex.

Findings also show that young men and women enter pre-marital relationships with differing expectations. Young women were considerably more likely than young men to have expected a romantic relationship to lead to marriage. Of those who reported a pre- marital romantic relationship, four in five young women $(79 \%)$ compared to one in two young men $(48 \%)$ intended to marry their romantic partner.

Progression of physical intimacy among youth who reported pre-marital romantic partnerships

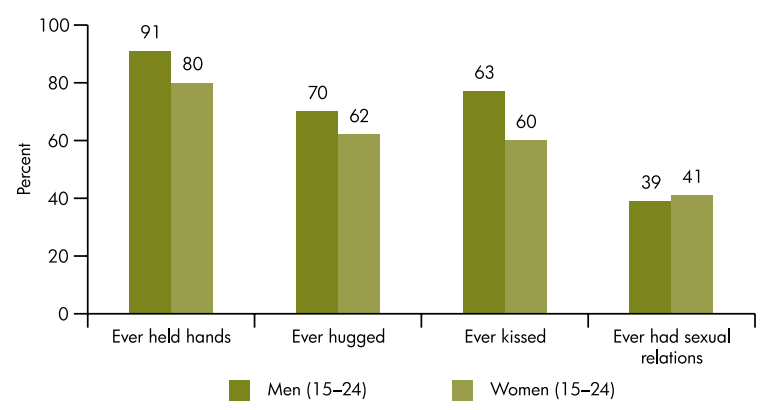

Sizeable numbers of young men and women engage in sex before marriage

The Youth Study inquired about pre-marital sex both with romantic partners and in other situations, for example, casual, paid and forced. Findings indicate that one in six young men $(17 \%)$ and slightly fewer than one in 10 young women $(7 \%)$ had engaged in pre-marital sex within romantic and/or other partnerships. Rural young men were twice as likely as their urban counterparts to have experienced pre-marital sex (19\% of rural young men compared to $10 \%$ of urban young men) and rural young women were four times more likely than their urban counterparts to report so ( $8 \%$ compared to $2 \%$ ).

\section{Extent of pre-marital sexual experiences among youth}

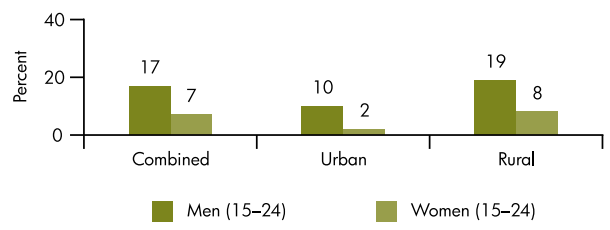

Many young people initiate pre-marital sex uninformed

Findings also confirm that many young people had initiated sexual activity uninformed.

For example, fewer than half of young men and women (45-46\%) who were sexually experienced before marriage knew that a woman can get pregnant at first sex.

In-depth awareness of contraceptive methods 
was also somewhat limited, particularly among sexually experienced young women. While $88 \%$ of young men who were sexually experienced before marriage had heard of condoms, fewer $(78 \%)$ knew that one condom can be used for only one sexual act. Among young women, the corresponding percentages were 55 and 31.

Likewise, awareness of sexually transmitted infections, including HIV/AIDS was limited among sexually experienced youth. While the majority of sexually experienced young men had heard of HIV (76\%), only $26 \%$ of young men reported comprehensive knowledge of HIV/AIDS ${ }^{1}$ and just $10 \%$ had ever heard of STIs other than HIV. The level of awareness was abysmally low among sexually experienced young women: just one in three had ever heard of HIV/AIDS; one in seven had comprehensive knowledge of HIV/AIDS and one in ten had ever heard of STIs other than HIV.
Condom use within pre-marital sexual relationships is almost non-existent

Consistent condom use was almost nonexistent. Among youth who had experienced pre-marital sex, only $7 \%$ of young men and $2 \%$ of young women reported that they had always used a condom.

Pre-marital sex is not always consensual Pre-marital sexual experience was nonconsensual for substantial minorities of

Of those who had experienced pre-marital sex, percentage of youth who reported multiple partnerships and consistent condom use

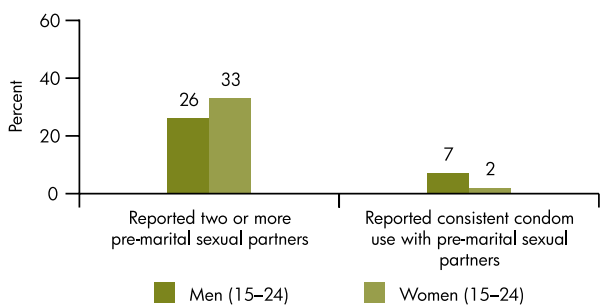

Of those who had experienced pre-marital sex, percentage of youth who reported in-depth awareness of sexual matters

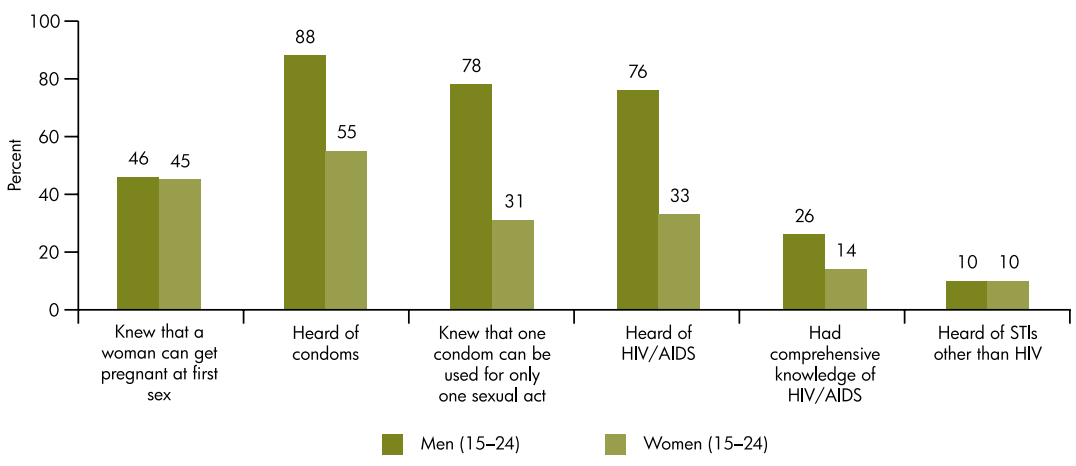

Engaging in pre-marital sex with multiple partners is not uncommon

Findings show that where pre-marital sex was experienced, it was by and large unsafe. Of those who were sexually experienced before marriage, over one-quarter of young men had sex with two or more partners. While fewer young women reported that they had engaged in pre-marital sexual relations, onethird of these young women reported multiple partners. young people, particularly young women. Of those who had engaged in pre-marital sexual relations, $6 \%$ of young men and as many as $24 \%$ of young women reported that they were forced to engage in sex. One in eight young men $(13 \%)$ and over two in five young women $(43 \%)$ reported that they were either persuaded or forced to engage in sex.

\section{Implications for programmes}

Findings presented above clearly highlight that many young people in Jharkhand had engaged

${ }^{1}$ Comprehensive awareness is defined as knowledge of two ways of preventing HIV (specifically, condom use and single partner relations), rejection of common misconceptions about HIV transmission (namely, that HIV can be transmitted through mosquito bites, sharing food or hugging) and awareness that one cannot tell by looking at a person whether he or she has HIV. 
Of those who had experienced pre-marital sex, percentage of youth who reported non-consensual sexual experiences

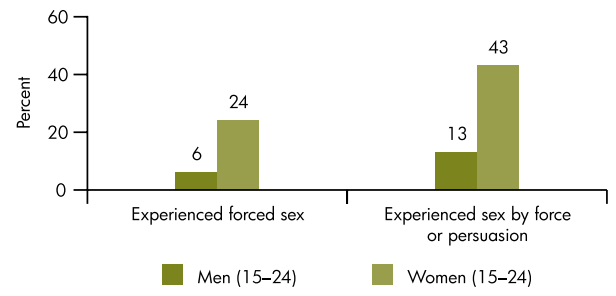

in sex before marriage and that many of them had initiated sexual activities uninformed, had engaged in unsafe relations and/or had experienced unwanted relations. Policies and programmes can no longer assume that pre-marital sex does not exist in our culture, rather they must ensure that sexual relations, should they occur, are informed, safe and wanted. Several steps are urgently needed.

Provide family life or sex education for those in school and out of school Youth must be equipped with information on physical maturation, relationship matters, risk and protective sexual behaviours (including the role of condoms). They must also be made aware of where they can obtain information, counselling and services. Sex and family life education programmes are needed that build awareness in ways that meet the needs of both those in school and out-of-school, responding to, rather than obfuscating, their questions on sexual health. Such information will allow youth to better assess whether to delay sexual relations, on the one hand and to ensure that relations, if undertaken, are safe, on the other.

Findings that even those in school had not been exposed to family life or sex education, notwithstanding the School AIDS Education Programme aimed at youth in Classes 9 and 11 in the state, emphasise the need for increased investment by the state in promoting such education for those in school and out of school.

Equally important is the acquisition of life skills that enable youth to put information into practice. Findings underscore the need for programmes that focus on building young people's skills in negotiating safe sex and communicating with partners, and that encourage young people to break down gender stereotypes and relate to each other as equals.

\section{Reorient service provision to address the sexual and reproductive health needs of unmarried youth}

At the same time, programmes must make available appropriate family planning and infection prevention services for unmarried young men and women in a manner acceptable to them. The National Adolescent Reproductive and Sexual Health strategy of the Reproductive and Child Health $(\mathrm{RCH})$ programme has recognised the need to address the needs of the unmarried, but much remains to be done in Jharkhand in terms of reorienting providers to include the unmarried in the ambit of services provided and to shed their own misgivings about addressing the needs of sexually active unmarried youth. 